\title{
Von Gottes Wort zum Ultraschall im ausserernsten Sinn
}

\author{
Jann Schwarzenbach \\ Dr. med., Facharzt für Allgemeinmedizin, FMH-Mitglied
}

An der Südspitze Apuliens, der antiken Finis Terrae, wo das Ionische Meer der Adria begegnet, thront auf einem Felsvorsprung die Wallfahrtskirche von Santa Maria di Leuca. Hier traf ich auf folgenden Anschlag: «Per pregare meglio, osserva il silenzio e spegni il telefonino.» Dass Geschwätz und Gezwitscher jede Andacht stören, erfordert keinen besonderen Kommentar, und ich wäre wohl achtlos an dem Hinweis vorbeigeschlendert, hätte die angenehme Kühle des Gotteshauses mich nicht zu einer besinnlichen Rast eingeladen. Der Name Leuca weist - gleichursprünglich mit unseren altvertrauten weissen Blutkörperchen - auf das griechische leukós hin, und eine gewisse Nähe zur Wiege der westlichen Kultur bezeugt auch ein schneller Blick aufs Smartphone: Es sucht sich in dieser

Küstengebiete waren seit jeher Orte der Begegnung und des Austausches. Das schuf auch neue geistige Strukturen.

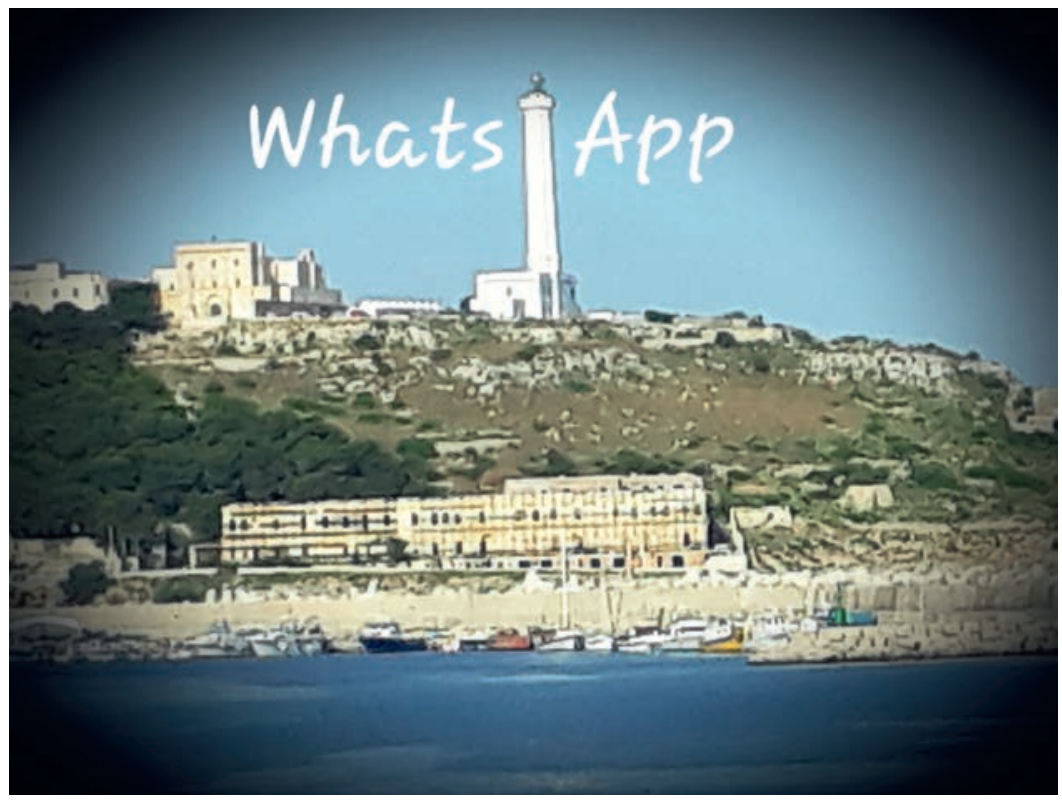

Gegend nämlich gerne einen hellenischen Netzanbieter. Küstengebiete waren seit jeher Orte der Begegnung und des Austausches, man lauschte dem weiten Meer und den Geschichten derer, die sich darauf hinausgewagt hatten. Das schuf auch neue geistige Strukturen, die unterschiedliche Denkweisen zu vereinen suchten. Dies gilt etwa für den Religionsphilosophen Philon von Alexandria (ca. 25 v.Chr. bis 40 n.Chr.), auf den ich weiter unten, im Zusammenhang mit der handynahen und gerade auch für die Medizin wichtigen Thematik des Hörens und des Sehens, etwas näher eingehen möchte.

\section{Zum Primat des Visuellen}

Im medizinischen Alltag sind Momente des entspannten und engagierten Zuhörens bekanntlich zur Mangelware geworden. Dies mag etliche Gründe haben, eine Mitschuld seitens der um sich greifenden Digitalisierung, und der damit verbundenen Fixation auf ubiquitäre Bildschirme, ist aber sicher nicht von der Hand zu weisen. Parallel dazu hat der Einsatz des Gehörsinns auch in der spezifischen Diagnostik zunehmend an Bedeutung verloren. So ist die Technik der Perkussion fast nur noch von historischem Interesse, und auch das Aufsetzen des Stethoskops, einst das standeseigene Werkzeug par excellence, wird immer mehr zum symbolischen Akt. Selbst die Anamnese basiert teilweise bereits auf vorbereiteten Formularen. Statt aufeinanderfolgenden Lautpaketen und Momenten der Stille sie sind auch ein Teil der Sprache - verarbeiten die medizinisch Tätigen hier algorithmisch aufgebaute, zeitgleich-visuell verfügbare Daten. Diese unterscheiden sich, was ihre Art betrifft, kaum von den - ohnehin übergewichteten - labor- und bildtechnisch erhobenen Befunden. Da zeigen sich eindeutige Parallelen zum Smartphone, mit dem ja kaum mehr telefoniert, sondern schriftlich oder lieber noch per Schnappschuss kommuniziert wird. Dabei ist das moderne Handy mehr als nur eine Weiterentwicklung traditioneller multimedialer Geräte. Das ins Netz gestellte Material hat nämlich direkten Aussagecharakter. Was auf 
dem Schirm erscheint, ist sozusagen die Stimme des Autors. In der Regel ist es kein Text, den man näher analysiert, kein Bild, das man distanziert betrachtet. Roh und ungefiltert erreichen die Daten im Direktzug via Sehbahn unser Inneres. Populisten aller Art haben das längst begriffen. Philon von Alexandria ebenfalls -wenn auch in einem ganz anderen Zusammenhang.

\section{Gottes Wort in deinem Ohr}

Der eben genannte Philosoph und Theologe entstammte einer einflussreichen jüdischen Familie und lebte in den Jahren um Christi Geburt im römisch besetzten, aber kulturell von Griechenland dominerten Alexandrien, wo nebst der ägyptischen Bevölkerung auch eine bedeutende israelitische Gemeinschaft angesiedelt war. Er studierte ebenso eifrig die Bibel wie auch die Werke der griechischen Philosophen und war von dem Gedanken fasziniert, in seinem kosmopolitischen Zuhause traditionellen Glauben mit dem Licht hellenischer Rationalität in Vereinbarung zu bringen.

Die Worte des Allmächtigen, die dann auf eine Art audiovisuellem Weg direkt das Innere unserer Seele zu erreichen vermögen.

So interpretierte er die Bibelinhalte, und die seiner Meinung nach darin verborgenen ewigen Wahrheiten, unter verschiedenen philosophischen Gesichtspunkten. Während in der religiösen Tradition die Auserlesenen des Schöpfers Stimme zu hören glaubten, setzten die griechischen Denker - als Zeichen zunehmender intellektueller Emanzipation und eines in Abbildern verstandenen Weltgefüges - eher auf visuelle Kanäle, um sich ihrem einzig Wahren und Guten anzunähern. Das Interesse unseres Denkers galt nun dem Versuch, die Leistungen von Auge und Ohr im Hinblick auf eine Begegnung mit dem himmlischen Herrscher miteinander zu vereinen. Er meinte begründen zu können, dass das Vernehmen von Gottes Stimme in Wirklichkeit ein visuell moduliertes Hören sei. Die Worte des Allmächtigen - man beachte hier die erstaunlichen anatomisch-physiologischen Kenntnisse - seien nämlich keine durch Gaumen und Zunge erzeugten Luftstösse, sondern leuchtende Manifestationen seines allumfassenden tätigen Geistes, die dann auf eine Art von audiovisuellem Weg direkt das Innere unserer Seele zu erreichen vermöchten.

\section{Zur Metaphysik des Schallkopfs}

Philons Verknüpfung von Theologie und Philosophie hat vor allem bei den frühchristlichen Denkern grosse Beachtung gefunden. Sein Werk umfasst Auseinandersetzungen mit verschiedensten geisteswissenschaftlichen Themen und erschöpft sich natürlich nicht in einer Theorie zur Sichtbarkeit von Gottes Wort. Gerade in der Ära des Smartphones hat aber die besonders intensive Wirksamkeit der visuellen Perzeption verführerischer Stimmen eine eigene und gefährliche Bedeutung gewonnen: Sie ist, wie bereits erwähnt, die Grundlage des Missbrauchs innerhalb der sozialen Medien. In diesem Zusammenhang sind auch wir digital vernetzte Ärzte und

Gerade in der Ära des Smartphones hat die intensive Wirksamkeit der visuellen Perzeption verführerischer Stimmen gefährliche Bedeutung gewonnen.

Ärztinnen - Stichwort: Stimme des Kommerzes - nicht vor Fallstricken verschiedenster Art gefeit. Über Philons Bedeutung für die heutige Medizin darf aber noch verwegener spekuliert werden: Auch die Sonographie basiert nämlich auf visuell modulierter Wahrnehmung akustischer Signale. Ob darin der tiefere Grund des durchschlagenden Erfolges dieser Untersuchungsmethode liegt, bleibe natürlich dahingestellt. Trotzdem könnte mit etwas Phantasie unser Philosoph durchaus als ihr Urvater bezeichnet werden. Wie dem auch sei: Sie hat - und das zu Recht - die vorgängige, auf dem Gehörsinn beruhende Perkussion in der täglichen Praxis ersetzt. Als orts- und patientennahe SlowTechnik lässt sie aber - im Gegensatz zu anderen bildgebenden Methoden - genügend Zeit zu direktem menschlichem Kontakt und könnte so dazu beitragen, den oft beklagten Bedeutungsverlust des Ab- und Zuhörens in der Medizin etwas zu entschärfen. Um schliesslich zum eingangs zitierten Hinweis in der Wallfahrtskirche zurückzukommen: Was wäre, wenn man zur stillen Andacht das Handy im Konferenzmodus eingeschaltet liesse? Würde der Betende da die Stimme seines Gottes sehen? Wohl kaum. Präsident Trumps latest Tweet aber schon.

Bildnachweis

Jann Schwarzenbach 